\title{
Trastorno por Déficit de Atención con Hiperactividad en Niños Escolarizados
}

\author{
ALFONSO URZÚA M. ${ }^{1}$, MARCOS DOMIC S. ${ }^{2}$, ANDREA CERDA C. ${ }^{2}$, \\ MIREYA RAMOS B. ${ }^{2}$, JAEL QUIROZ E. ${ }^{2}$ \\ 1. Psicólogo, Magíster en Salud Pública, Doctor en Psicología Clínica y de la Salud. Área Psicología de la Salud, Escuela de \\ Psicología, Universidad Católica del Norte. \\ 2. Psicólogo, Licenciado en Psicología. Área Psicología de la Salud, Escuela de Psicología, Universidad Católica del Norte.
}

\begin{abstract}
Attention Deficit/Hyperactivity Disorder (ADHD) in School Age Children in Antofagasta

Objective: To evaluate the prevalence of Attention Deficit/Hyperactivity Disorder (ADHD) in the city of Antofagasta. Patients and Methods: Teachers and parents of 640 children (290 boys and 350 girls) between 6 and 11 years, from public, subsidized and private schools were evaluated through the Attention Deficit Hyperactivity Disorder Rating Scale-IV [ADHD-RS IV]. Results: Utilizing the ADHD Scale independently, the prevalence ranged between $5 \%$ and $15 \%$ depending on the informant. This number decreased to $2 \%$ when the criteria were required both at home and school environments. Conclusion: In our population the prevalence of ADHD is highest in boys 6 to 8 years of age, being the combined disorder the most common subtype found in this group.
\end{abstract}

(Key words: Attention Deficit Hyperactivity Disorder, Prevalence, ADHD RS-IV).

Rev Chil Pediatr 2009; 80 (4): 332-338

\section{RESUMEN}

Objetivo: Describir la prevalencia encontrada del Trastorno por déficit de atención con hiperactividad en la ciudad de Antofagasta. Método: A través de las Escalas Attention Deficit Hiperactivity Disorder Rating Scale-IV [ADHD RS-IV] fueron evaluados apoderados y profesores de 640 niños (290 niños y 350 niñas) entre 6 y 11 años, provenientes de establecimientos educacionales públicos, subvencionados y privados. Resultados: Se encuentran prevalencias que oscilan entre un 5\% a un $15 \%$ dependiendo del informante, las cuales disminuyen a un $2 \%$ considerando criterios cumplidos en dos ambientes. Existen diferencias significativas entre hombres y mujeres, así como entre rangos de edad. Conclusión: La prevalencia estimada de TDAH es mayor en hombres entre 6 a 8 años, siendo el subtipo más frecuente el combinado. (Palabras clave: Trastorno por déficit de atención con hiperactividad, Prevalencia, ADHD RS-IV). Rev Chil Pediatr 2009; 80 (4): 332-338

Trabajo recibido el 18 de marzo de 2009, devuelto para corregir el 24 de abril de 2009, segunda versión el 14 de mayo de 2009, aceptado para publicación el 28 de junio de 2009.

Correspondencia a:

Dr. Alfonso Urzúa M.

E-mail: alurzua@ucn.cl 


\section{Introducción}

El Plan nacional de Salud Mental y Psiquiatría del Ministerio de Salud de Chile ${ }^{1}$ considera el Trastorno por Déficit Atencional con Hiperactividad (TDAH) como el problema de salud mental más frecuente en niños, niñas y adolescentes en edad escolar en Chile, estimándose que uno de cada 80 a 100 escolares de enseñanza básica requieren tratamiento específico por dicho trastorno, siendo el diagnóstico neurológico más frecuente en los servicios de atención primaria ${ }^{2}$.

El TDAH es definido como un trastorno conductual que surge en la infancia teniendo como característica fundamental un patrón persistente de desatención y/o hiperactividad e impulsividad presentes en al menos dos contextos. De acuerdo al predominio de síntomas que estén presentes, el Trastorno por déficit de atención con hiperactividad (TDAH) se clasifica en tres subtipos: con predominio de déficit de atención, con predominio hiperactivo/impulsivo y subtipo combinado ${ }^{3}$.

En términos epidemiológicos, a nivel internacional, las tasas de prevalencia del TDAH son discrepantes. En Estados Unidos se estiman prevalencias que van desde un 3 a un $5 \%^{3}$, a diferencia de Europa, donde se obtienen prevalencias de $1,5 \%{ }^{4}$.

En numerosos estudios se ha estimado la prevalencia del TDAH en distintas poblaciones obteniendo tasas diversas, por ejemplo: $4,7 \%$ en niños de 6 a 11 años en Mallorca ${ }^{5} ; 10,15 \%$ en niños venezolanos entre 3 y 13 años de Maracaibo $^{6} ; 16,1 \%$ en niños colombianos entre 4 y 17 años de Manizales y un 18\% en niños entre 6 a 11 años en Medellín ${ }^{7,8}$. En población general DuPaul et $\mathrm{al}^{9}$, han referido un intervalo entre un 2 y $30 \%$ y Buitelaar \& Van Engeland entre un 4 y $17 \%{ }^{10}$.

El elevado grado de variabilidad de tasas de prevalencia se ha explicado por los distintos métodos de evaluación utilizados, la diversidad y cambios de los criterios clínicos diagnósticos, diferentes informantes (padres, profesores o cuidadores/tutores), el tipo de muestra escogida (clínica o poblacional) o por características sociodemográficas diversas ${ }^{5,10,11}$.

En Chile, se han realizado diversas investi- gaciones en el área del TDAH ${ }^{12-14}$, sin embargo, son pocas las que presentan estimaciones de prevalencia. En la literatura indexada sólo se ha encontrado referencias de tres estudios: a) en segundo año básico en Talca ${ }^{15}$, quienes reportan una prevalencia cercana al $18 \%$ pero evaluados a través de sólo un informante; b) en población aymará ${ }^{16}$, cuyo estudio igualmente se centra en datos recogidos de un sólo informante y c) un estudio de prevalencia realizado en Chillán ${ }^{17}$. En este último la población del estudio comprendió preescolares entre 3 a 5 años, período evolutivo no recomendado para efectuar un diagnóstico de TDAH, dado que las características evolutivas propias de la edad preescolar así como su desarrollo neurobiológico no permite claridad en diagnóstico ${ }^{18}$. A esa edad la corteza prefrontal y las funciones ejecutivas como la inhibición de la respuesta y control motor aún no se encuentran bien desarrolladas $^{19}$. Así como también lo señala el DSMIV-TR, en su criterio B para el diagnóstico, que los síntomas de hiperactividad/impulsividad deben haber estado presente antes de los 7 años de edad, sugiriendo esperar hasta la edad escolar para entregar un diagnóstico más preciso ${ }^{3}$.

El objetivo de este estudio es describir la prevalencia estimada del TDAH en población escolarizada de seis a once años en la ciudad de Antofagasta.

\section{Pacientes y Método}

\section{Participantes}

Se consideró una muestra no probabilística e intencionada, cuidando una distribución similar para rangos de edad y sexo, de 640 participantes entre 6 a 11 años de edad. Docientos veintiocho de ellos $(35,6 \%)$ acudían a establecimientos municipales, $200(31,3 \%)$ a subvencionados y 212 a establecimientos particulares $(33,1 \%)$. Los cuestionarios fueron completados por 612 apoderados y 82 profesores. La tasa de respuesta fue entre un 75 y un $100 \%$ dependiendo del establecimiento en el caso de los profesores y de un $85 \%$ para los apoderados.

Se reportan prevalencias estimadas por sexo y edad, dado que se han reportado diferencias en estas dadas por ambos factores ${ }^{3,7,8,11,17,20-22}$. 
El nivel socioeconómico no se considerará como variable porque su factor de riesgo relativo es tan sólo de un $1,15^{23}$ y ha presentado resultados discrepantes en estudios de evaluación del $\mathrm{TDAH}^{7,17}$.

\section{Instrumento}

Se utilizó la Attention Deficit Hiperactivity Disorder Rating Scale-IV [ADHD RS-IV] ${ }^{24}$, adaptada a población española ${ }^{22}$. Esta se compone de 18 ítems que coinciden con los síntomas pertenecientes al criterio A del DSM-IV para el diagnóstico de TDAH. Consta de una subescala de Inatención, otra de Hiperactividad/ Impulsividad y la escala total. La escala ha presentado buenas propiedades psicométricas tanto en España ${ }^{22}$ como en Estados Unidos ${ }^{25}$ y en Chile, en donde las dimensiones evaluadas mostraron una consistencia interna entre ,76 y ,90 en la escala para apoderados (,92 para la escala total) y entre, 95 y ,97 en la versión para profesores (,97 para la escala total) (Urzúa A, Domic M, Ramos M, Cerda A \& Quiroz J. Evaluación de escalas para el diagnóstico del Trastorno por déficit de atención con hiperactividad en Chile. Artículo no publicado).

\section{Procedimiento}

Se seleccionaron establecimientos educacionales intentando incorporar tanto establecimientos de tipo públicos, como subvencionados y privados, a los cuales se solicitó la autorización para realizar la investigación. Una vez obtenidos los permisos correspondientes, la aplicación se realizó en las reuniones de apoderados en al menos un curso por nivel por cada establecimiento. En cada curso se solicitaba la colaboración a los siete primeros apoderados en llegar a la reunión. A su vez, se les solicitó a los profesores jefe de cada curso, contestar las escalas para valorar la conducta de los menores evaluados por los apoderados. El motivo de la selección de siete menores por curso responde a la necesidad de evitar un exceso en la cantidad de escalas rellenadas por cada profesor. Una vez recogidos los cuestionarios, estos fueron ingresados a una base de datos construida en SPSS 16.0.

\section{Análisis estadísticos}

Se realizan distribuciones de frecuencia de los distintos subtipos de TDAH a nivel global, como estratificando por sexo y rango de edad. Se realizan tres análisis, primero considerando los informes solamente completados por los apoderados, segundo los completados por los profesores, y un tercer análisis en donde se analizaron los cuestionarios para cada sujeto por ambos informantes, considerando así el criterio de dos ambientes requerido para el diagnóstico ${ }^{3}$. El punto de corte establecido para cada uno de los subtipos fue de 6 conductas de 9 para cada factor, de acuerdo a lo esperado en el DSM-IV.

\section{Resultados}

\section{Participantes}

De total de 640 sujetos evaluados, 299 tenían entre 6 a 8 años $(46,7 \%)$ y $341(53,3 \%)$ entre 9 y 11 años. La media de edad de la muestra total fue de 8,76 años (DT =1,78). La distribución de participantes por sexo, edad y tipo de establecimiento al cual acudían se encuentra en la tabla 1.

\section{Prevalencias Estimadas}

La tabla 2 nos muestra la prevalencia estimada según la evaluación realizada por los

Tabla 1. Distribución de la muestra según sexo, rango de edad y tipo de establecimiento (E)

\begin{tabular}{|c|c|c|c|c|c|c|c|c|c|c|c|c|c|c|c|c|}
\hline \multirow{3}{*}{ Edad } & \multicolumn{4}{|c|}{ E. Público } & \multicolumn{4}{|c|}{ E. Subvencionado } & \multicolumn{4}{|c|}{ E. Particular } & \multicolumn{4}{|c|}{ Total } \\
\hline & \multicolumn{2}{|c|}{ Hombre } & \multicolumn{2}{|c|}{ Mujer } & \multicolumn{2}{|c|}{ Hombre } & \multicolumn{2}{|c|}{ Mujer } & \multicolumn{2}{|c|}{ Hombre } & \multicolumn{2}{|c|}{ Mujer } & \multicolumn{2}{|c|}{ Hombre } & \multicolumn{2}{|c|}{ Mujer } \\
\hline & n & $\%$ & $n$ & $\%$ & $n$ & $\%$ & n & $\%$ & $n$ & $\%$ & n & $\%$ & $n$ & $\%$ & n & $\%$ \\
\hline $6-8$ & 50 & 47,2 & 56 & 52,8 & 46 & 50 & 52 & 48,1 & 39 & 45,3 & 56 & 44,4 & 135 & 46,6 & 164 & 46,9 \\
\hline $9-11$ & 62 & 50,8 & 60 & 49,2 & 46 & 50 & 56 & 51,9 & 47 & 54,7 & 70 & 55,6 & 155 & 53,4 & 186 & 53,1 \\
\hline Total & 112 & 116 & 116 & 100 & 92 & 100 & 108 & 100 & 88 & 100 & 126 & 100 & 290 & 45,3 & 350 & 54,7 \\
\hline
\end{tabular}


apoderados. Tal como se observa, esta es cercana a un $6 \%$ en la muestra total, existiendo diferencias al estratificar por sexo, siendo mayor en los hombres $(7,1 \%)$ que en las mujeres $(4,6 \%)$ y en el rango de menor edad $(7,2 \%)$ por sobre el de 9 a 11 años (4,3\%). Tanto en los hombres como en el grupo de menor edad predomina el TDAH del subtipo combinado.

Cuando los que evalúan son los profesores, la prevalencia estimada aumenta en todos los grupos de análisis con relación a la prevalencia percibida por los apoderados: a nivel global $(14,9 \%)$, en los hombres $(22,3 \%)$, las mujeres $(8,2 \%)$, en el rango de 6 a 8 años $(20,1 \%)$ y de 9 a 11 años $(10,1 \%)$. Sigue siendo mayor la prevalencia reportada para los hombres y el grupo de 6 a 8 años (tabla 3 ).
Al considerar la evaluación que para el mismo sujeto realizan tanto el apoderado como el profesor, es decir, cumpliendo con los criterios DSM-IV de presentar conductas en a lo menos dos ambientes, se encuentra que la prevalencia disminuye notablemente a un $1,3 \%$ a nivel total, aumentando ligeramente a un $2,9 \%$ en los hombres y un $2,7 \%$ en el grupo de 6 a 8 años (tabla 4).

Cabe destacar que al considerar los sujetos que cumplían con los criterios por separado en ambos ambientes, pero cuya clasificación fue de distintos subtipos por cada informante (profesor clasifica inatento y apoderado hiperactivo), la prevalencia aumentaría, ya que es posible encontrar en esta categoría 5 (aumentando a un $2,5 \%$ la prevalencia total). De estos 5 casos

Tabla 2. Prevalencia estimada por los apoderados según sexo, rango de edad y total de la muestra

\begin{tabular}{|c|c|c|c|c|c|c|c|c|c|c|}
\hline & \multicolumn{2}{|c|}{ Hombre } & \multicolumn{2}{|c|}{ Mujer } & \multicolumn{2}{|c|}{ 6-8 años } & \multicolumn{2}{|c|}{ 9-11años } & \multicolumn{2}{|c|}{ Total } \\
\hline & Frec & $\%$ & Frec & $\%$ & Frec & $\%$ & Frec & $\%$ & Frec & $\%$ \\
\hline Sin trastorno & 250 & 92,9 & 314 & 95,4 & 256 & 92,8 & 308 & 95,7 & 564 & 94,3 \\
\hline TDAH subtipo inatento & 6 & 2,2 & 3 & 9 & 6 & 2,2 & 3 & 9 & 9 & 1,5 \\
\hline TDAH subtipo hiperactivo impulsivo & 5 & 1,9 & 8 & 2,4 & 5 & 1,8 & 8 & 2,5 & 13 & 2,2 \\
\hline TDAH subtipo combinado & 8 & 3,0 & 4 & 1,2 & 9 & 3,3 & 3 & 9 & 12 & 2,0 \\
\hline Total & 269 & 100,0 & 329 & 100,0 & 276 & 100,0 & 322 & 100,0 & 598 & 100,0 \\
\hline
\end{tabular}

Tabla 3. Prevalencia estimada por los profesores según sexo, rango de edad y muestra total

\begin{tabular}{|c|c|c|c|c|c|c|c|c|c|c|}
\hline & \multicolumn{2}{|c|}{ Hombre } & \multicolumn{2}{|c|}{ Mujer } & \multicolumn{2}{|c|}{ 6-8 años } & \multicolumn{2}{|c|}{ 9-11años } & \multicolumn{2}{|c|}{ Total } \\
\hline & Frec & $\%$ & Frec & $\%$ & Frec & $\%$ & Frec & $\%$ & Frec & $\%$ \\
\hline Sin trastorno & 192 & 77,7 & 247 & 91,8 & 198 & 79,8 & 241 & 89,9 & 439 & 85,1 \\
\hline TDAH subtipo inatento & 21 & 8,5 & 7 & 2,6 & 20 & 8,1 & 8 & 3,0 & 28 & 5,4 \\
\hline TDAH subtipo hiperactivo impulsivo & 13 & 5,3 & 6 & 2,2 & 14 & 5,6 & 5 & 1,9 & 19 & 3,7 \\
\hline TDAH subtipo combinado & 21 & 8,5 & 9 & 3,3 & 16 & 6,5 & 14 & 5,2 & 30 & 5,8 \\
\hline Total & 247 & 100,0 & 269 & 100,0 & 248 & 100,0 & 268 & 100,0 & 516 & 100,0 \\
\hline
\end{tabular}

Tabla 4. Prevalencia estimada considerando ambos informantes según sexo, rango de edad y para la muestra total

\begin{tabular}{|c|c|c|c|c|c|c|c|c|c|c|}
\hline & \multicolumn{2}{|c|}{ Hombre } & \multicolumn{2}{|c|}{ Mujer } & \multicolumn{2}{|c|}{ 6-8 años } & \multicolumn{2}{|c|}{ 9-11años } & \multicolumn{2}{|c|}{ Total } \\
\hline & Frec & $\%$ & Frec & $\%$ & Frec & $\%$ & Frec & $\%$ & Frec & $\%$ \\
\hline Sin trastorno & 170 & 97,1 & 224 & 100 & 177 & 97,3 & 217 & 100 & 394 & 98,7 \\
\hline TDAH subtipo inatento & 2 & 1,1 & & & 2 & 1,1 & & & 2 &, 5 \\
\hline TDAH subtipo hiperactivo impulsivo & 1 &, 6 & & & 1 &, 5 & & & 1 &, 3 \\
\hline TDAH subtipo combinado & 2 & 1,1 & & & 2 & 1,1 & & & 2 &, 5 \\
\hline Total & 175 & 100,0 & 224 & 100 & 182 & 100,0 & 217 & 100 & 399 & 100,0 \\
\hline
\end{tabular}


4 eran hombres (aumento a un 5\% de la prevalencia en hombres) y 4 del rango de edad de 6 a 8 años (aumentando a un 4,8\% la prevalencia para ese tramo etáreo).

\section{Discusión}

Las prevalencias encontradas en esta muestra difieren de las encontradas en otros estudios fundamentalmente al considerar el cumplimiento de los criterios diagnósticos en a lo menos dos ambientes.

En la literatura se ha reportado discrepancias como la observada en esta investigación, sobre las diferencias que se suelen encontrar basadas en el tipo de informante. En España se realizó un estudio contrastando los tipos de informante en la escala ADHD RS-IV presentándose diferencias contrarias a las encontradas en esta investigación, en donde los apoderados mostraron medias superiores a los profesores $^{22}$. Para este fenómeno es posible considerar distintos factores ambientales tales como las condiciones culturales y educacionales en las cuales se enmarca el contexto español, las que distanciarían los resultados obtenidos en la población chilena, en donde son los profesores quienes reportan una mayor cantidad de niños con posible TDAH. Cabe destacar que el porcentaje encontrado en esta investigación al considerar sólo el informe del profesor, es similar a lo reportado por Verdugo en el estudio de Talca ${ }^{15}$, sin embargo, con la evaluación de un sólo ambiente no se cumple los criterios diagnósticos requeridos.

Posibles explicaciones son la cantidad de niños (as) presentes en cada sala, la mayor cantidad de horas que pasan los niños en el aula escolar que posibilitaría una mayor cantidad de horas de observación, la dificultad en los padres de reconocer algún posible problema en los menores o la expectativa del profesor de que si se evalúa mal a los menores, se tomarán medidas para controlar las conductas de éstos.

Pese a esta discrepancia, aun es posible observar un porcentaje de alrededor de un $2 \%$ que cumple con los criterios para ambos informantes, llegando incluso a un 5\% si se consideran las disparidades en la clasificación en los distintos subtipos. Esto puede ser debido a que es más probable que un padre observe con mayor facilidad en el hogar conductas vinculadas a la inatención, en comparación con los profesores, quienes podrían centrar más su atención en la presencia de conductas hiperactivas, en especial cuando estas provocan irrupción en el aula.

En cuanto a las diferencias dadas por el sexo, se logran observar diferencias entre niños y niñas, en la que los niños obtienen puntajes más altos, lo que se condice con los resultados obtenidos en otras investigaciones ${ }^{3,8,11,20,22}$. En 1999, un estudio apoyado por el Instituto Nacional de Salud Mental estadounidense ${ }^{26}$ encontró que existen diferencias entre sexo en la forma clínica como afecta el TDAH a mujeres y hombres, principalmente en la mayor posibilidad por parte de las mujeres de presentar síntomas de inatención en comparación a los hombres en quienes predominan los síntomas de hiperactividad, impulsividad y comportamientos disruptivos secundarios.

Estas diferencias se podrían relacionar a la manifestación conductual del TDAH, ya que los síntomas que más se logran percibir tanto en el contexto familiar como escolar son los referidos a la hiperactividad e impulsividad debido a lo disruptivo de su naturaleza a diferencia de los síntomas inatentos los cuales desde la normativa social es esperable que las mujeres presenten conductas pasivas, por lo que el modelado y estilo de crianza proporcionado por los padres, irían en tal dirección.

De acuerdo a la edad, los resultados obtenidos apuntan a una diferencia entre las edades de 6 a 8 años respecto a los niños de 9 a 11 siendo mayor en el primer grupo los síntomas de hiperactividad e impulsividad, es decir a medida que los niños crecen, las conductas hiperactivas disminuirían progresivamente gracias al desarrollo evolutivo, dado que los niños logran adaptarse mejor a su trastorno y son más capaces de inhibir conductas inapropiadas $^{11}$.

Dentro de la investigación es posible encontrar dificultades asociadas en cuanto al acceso a la muestra, en relación a las restricciones horarias en las cuales era posible contactar a los apoderados y las limitaciones relativas al 
contexto de una reunión de apoderados. Esto y factores externos asociados al contexto educacional como movilizaciones, reglamentación de cada establecimiento y actividades propias de los recintos de aplicación, concluyeron en una demora en la obtención de la muestra, requiriendo utilizar un período de tiempo mayor a lo esperado.

Otra dificultad que se presentó en este estudio, es que el tipo de informante "apoderado" incluye la evaluación de madres, padres, abuelos o tutores del menor, lo cual constituye una variable que puede incidir en las puntuaciones realizadas por lo que se sugiere considerar esta diferencia en futuras investigaciones.

La cantidad de la muestra en la presente investigación no permite realizar baremaciones, por lo que se sugieren posteriores estudios con muestras mayores.

\section{Referencias}

1.- Minsal: Ministerio de Salud de Chile. Plan Nacional de Salud Mental y Psiquiatría. Ediciones Unidad de Salud Mental. 2 ${ }^{\text {a }}$ Edición, Chile. 2001.

2.- Lavados P, Gómez V, Sawada M, Chomali M, Álvarez $M$ : Diagnósticos neurológicos en la atención primaria de salud en Santiago, Chile. Rev Neurol 2003; 36 (6): 518-22.

3.- American Psychiatric Association: Trastorno por déficit de atención con hiperactividad. Manual diagnóstico y estadístico de los trastornos mentales. Editorial Masson S.A. España. 2005.

4.- Taylor E, Döpfner M, Sergeant J, Ashersonm P: European clinical guidelines for hyperkinetic disorderfirst upgrade. Eur Child Adolesc Psychiatr 2004; Supl 1: S7-30.

5.- Cardó E, Servera M, Llobera J: Estimación de la prevalencia del trastorno por déficit de atención e hiperactividad en población normal de la isla de Mallorca. Rev Neurol 2007; 44 (1): 10-4.

6.- Montiel-Nava C, Peña J, Montiel-Barbero I: Datos epidemiológicos del trastorno por déficit de atención con hiperactividad en una muestra de niños marabinos. Rev Neurol 2003; 37 (9): 815-9.

7.- Pineda D, Henao G, Puerta I, et al: Uso de un cuestionario breve para el diagnóstico de deficiencia atencional. Rev Neurol 1999; 28 (4): 365-72.

8.- Pineda D, Lopera F, Henao G, Palacio J, Castellanos F, Grupo de Investigación Fundema: Confirma- ción de la alta prevalencia del trastorno por déficit de atención en una comunidad colombiana. Rev Neurol 2001; 32 (3): 1-6.

9.- DuPaul G, Power JT, Anastopoulos AD, Reid R: ADHD-Rating Scales DSM-IV for parents and teachers. New York: Guilford Press; 1998.

10.- Buitelaar JK, Van Engeland H: Epidemiological approaches. In Sandberg S, ed. Hyperactivity disorders of childhood. Cambridge: Cambridge University Press; 1996.

11.- Blázquez G, Joseph D, Burón E, et al: Resultados del cribado de la sintomatología del trastorno por déficit de atención con o sin hiperactividad en el ámbito escolar mediante la escala EDAH. Rev Neurol 2005; 41 (10): 586-90.

12.- Campos C, Fernández P, Mobarec S, Claro S, Sánchez I: Relación entre el síndrome de apnea obstructiva del sueño y el trastorno de déficit atencional con hiperactividad. Estudio en una población de escolares chilenos. Rev Chil Pediatr 2003; 74 (1): 46-52.

13.- Carrasco $X$, Rothhammer P, Moraga M, Henríquez $H$, Aboitiz F, Rothhammer F: Presencia de los alelos DRD4/7R y DAT1/10R en miembros de familias chilenas con síndrome de déficit atencional con hiperactividad. Rev Méd Chile 2004; 132: 1047-52.

14.- Valdivieso A, Cornejo A, Sánchez M: Tratamiento del síndrome de déficit atencional (SDA) en niños: evaluación de la moclobemida, una alternativa no psicoestimulante. Rev Chil Neuro-Psiquiatr 2000; 38 (1): 7-14.

15.- Verdugo L, Astaburuaga M, Muñoz C, Navarrete D: Test de Conners en niños de segundo básico de la provincia de Talca. Rev Chil Pediatr 2006; 77 (6): 621-88.

16.- Rothhammer P, Carrasco X, Henriquez H, et al: Bajo riesgo de déficit atencional/hiperactividad en niños aymarás: Implicancias genéticas, antropológicas y culturales. Chungará 2005; 37 (2): 145-9.

17.- Herrera $G$ : Prevalencia del déficit atencional con hiperactividad en nños de 3 a 5 años de edad de la ciudad de Chillán, Chile. Theoria 2005; 14 (2): 45-55.

18.- Cavallo V, Simón M: Manual de psicología clínica infantil y del adolescente. Trastornos específicos. Madrid: Pirámide. 2002.

19.- Martínez A, Moreno D, Soler B: Evaluación del diagnóstico y tratamiento de los niños con trastorno por déficit de atención/hiperactividad en España mediante la técnica Achievable Benchmarks of Care (ABC). Rev Neurol 2008; 47 (9): 451-6.

20.- Oades R, Sadile A, Sagvolden T, et al: The control 
of responsiveness in ADHD by catecholamines: evidence for dopaminergic, noradrenergic and interactive roles. Developmental Science 2005; 8 (2): 122-31.

21.- DuPaul G, Weyandt L: School-Based Interventions for children and adolescents with Attention-deficit/ hiperactivity disorder: Enhancing academia and behavioral outcomes. Education and treatment of children 2006; 29 (2): 341-58.

22.- Servera $M$, Cardó E: ADHD Rating Scale-IV en una muestra escolar española: datos normativos y consistencia interna para maestros, padres y madres. Rev Neurol 2007; 45 (7): 393-9.

23.- Soutullo C, Diez A: Manual de diagnóstico y trata- miento del TDAH. Madrid: Editorial Médica Panamericana. 2007.

24.- DuPaul GJ, Power TJ, Anastopoulos AD, et al: Teacher rating of attention deficit hyperactivity disorder symptoms: Factor structure and normative data. Psychological Assessment 2007; 9: 436-43.

25.- Myers K, Collett B: Escalas de evaluación. En Wiener J, Dulcan M. (Eds) Tratado de Psiquiatría de la infancia y la adolescencia. España: Masson. 2006.

26.- Biederman: Características y variabilidad clínica del trastorno por déficit de atención/hiperactividad en niñas, 1999. En Valdizán J, Mercado E, Mercado A. Rev Neurol 2007; 44 (2): 27-30. 